\title{
Human preferences and risky choices
}

\author{
Paul van Schaik ${ }^{1}$, Petko Kusev ${ }^{2 *}$ and Asgeir Juliusson ${ }^{3+}$ \\ Department of Psychology, Teesside University, Middlesbrough, UK \\ 2 Department of Psychology, Kingston University London, London, UK \\ ${ }^{3}$ Department of Psychology, School of Social Sciences, City University, London, UK \\ *Correspondence: p.kusev@kingston.ac.uk \\ ${ }^{\dagger}$ Deceased
}

There are different views on what preferences for risks are and whether they are indicators of stable, underlying generic cognitive systems. Preferences could be conceived as an attitude toward a set of properties of context, memory, and affect - a gage of how much uncertainty one is willing to tolerate. One type of computational "descriptive" integrative decision-making theories predicts specific behavioral patterns of risky preferences. An individual's risky choice among two or more options is considered, where at least one option has an uncertain outcome ${ }^{1}$. Choices are based on the integration of probability and utility information into expected utilities, and tradeoff comparisons of computed outcomes. It is assumed that there are lawful underlying patterns of risky preferences (e.g., the shapes of loss aversion and probability-weighting functions), and that these would reflect any relevant constraints in cognitive resources. In this spirit, in this research topic, Lebière and Anderson demonstrate that their sequence-learning model, reflecting general cognitive processes in response to constraints inherent in the task environment, is superior for modeling risky choice in terms of capturing the stability that comes from previous experience. According to Luce, there are three inherently different types of people corresponding to their values of an additional utility-model parameter representing risk preference. Birnbaum demonstrates that the TAX model, in contrast to other explanations, accounts for a lack or transitivity in people's choices. Pothos and Busemeyer show that quantum-probability theory allows the modeling of decision-making phenomena (e.g., the conjunction fallacy and violations of the sure-thing principle), which go beyond classic probability theory, because of the context- and order-dependence in quantum-probability assessment. Jones and Oaksford provide evidence for a more stable pattern of preferences in transactional decision tasks than in gambles. Given that hypothetical gambles provide results that are internally inconsistent, Baron demonstrates that a monetary-difference choice task to measure risk preference is a good indicator of people's utility function for money.

Another type of theory can be considered as "noncomputational." These theories argue for processing by establishing the role of "experience" in risky decision-making, proposing that choices are not based on the utilitarian integration of probability, and utility information, and trade-off comparisons of computed outcomes. However, yet (again) it is assumed that there are lawful underlying patterns of preferences, or people use specific processing and decision-making strategies. Stewart's results of model fitting show that, for simple risky choices, an additive ("non-integrative")

${ }^{1}$ We use the terms "decision-making" and "choice" interchangeably. We prefer "choice," as it more clearly indicates individual behavior, but we use "decision-making," "decision," or "decision-maker" when linguistically it appears more natural to do so. model can completely mimic a multiplicative ("integrative") model; however, even stability of parameter values over time and across contexts in the different models does not imply correct model identification, as the parameters map onto different psychological variables. Betsch argues and provides evidence for the conceptualization of preferences as attitudes, whose stability is determined by behavior repetition and processing style. According to Hertwig and Gigerenzer, apparent inconsistencies in risky-choice behavior can be accounted for by decision-makers' application of cognitive strategies (in particular heuristics) and the interaction of these strategies with the environment. Brandstätter contends that elicitation method strongly affects people's choices; people use many strategies, one main candidate of which is the priority heuristic. Parducci demonstrates that range-frequency theory implies that judgments are not stable across contexts; as a result, the search for higher utility leads to reduced pleasure. Brown and Matthews show that, at least under certain conditions, rank-based models and range-based models are equivalent in that both can account for apparent range effects.

Yet, still other authors explore arguments for a moderation of computational and non-computational processes of decision-making by other factors. They highlight the possibility that memory or experiences of events leak into decisions even when risk information is explicitly provided. In this research topic, Kusev and van Schaik argue and provide evidence for the idea that characteristics of (a) the decision-making context and (b) content, (c) the decision-maker (including cognitive resources and motivation), and (d) presentation format of task material (for example probability format or frequency format) all influence people's psychological processing and subsequent risky choices. It follows then that stable behavioral patterns toward risk or the use of (single) psychological strategies do not exist. Chater, Johansson, and Hall also argue that people do not have risk preferences; rather, risky choices are shaped directly by past choices or explanations thereof. Any coherence between choices will be limited to those that share superficial features.

Still other researchers provide further accounts for the apparent lack of stability of preferences. In this research topic, Fox and Tannenbaum argue that because of four specific conceptual and methodological challenges there is still a lack of evidence for stable and measurable risk preferences. Aldrovandi and van Heussen argue that the lack or degree of stability of preference in decisionmaking can be explained by psychological phenomena of memory; various memory phenomena lead to instability of risk preferences. Based on evidence from their neuropsychological brain research, Chen, Allen, Deb, and Humphreys argue that emotions can play a necessary functional role in decision-making, but as a consequence, emotions can alter the stability of the process. According to Dickert 
and Slovic, research on mental imagery and attention as underlying processes of affective responses and other research showing individual differences as moderators of these processes help explain why people do not hold stable values for saving human lives. Vlaev shows and provides evidence for the idea that trade-off inconsistency is a ubiquitous psychophysical anomaly, in which preferences between (pairs of) options are not reliable when the options are of the same qualitative type and/or differ on a single dimension. Villejoubert and Vallée-Tourangeau argue that the perspective of distributed cognition has the potential to provide a new way of conceiving of and accounting for the role of the environment in the construction of preference; the implication is that preferences may be very different when people interact with rather than respond to the environment.
In conclusion, the contributions in this research topic offer a range of explanations for stability in risky choice. We are looking forward to further work that comparatively tests the validity of these different explanations and work that integrates approaches to provide a better account where this seems is appropriate.

Received: 26 October 2011; accepted: 26 October 2011; published online: 15 November 2011.

Citation: van Schaik P, Kusev P and Juliusson A (2011) Human preferences and risky choices. Front. Psychology 2:333. doi: 10.3389/fpsyg.2011.00333

This article was submitted to Frontiers in Cognition, a specialty of Frontiers in Psychology. Copyright $\odot 2011$ van Schaik, Kusev and Juliusson. This is an open-access article subject to a non-exclusive license between the authors and Frontiers Media SA, which permits use, distribution and reproduction in other forums, provided the original authors and source are credited and other Frontiers conditions are complied with. 